\title{
Gastroparesia diabética: revisión corta con viñeta clínica ilustrativa. Actualización y puntos más relevantes para la práctica clínica
}

\author{
Diabetic gastroparesis: Short review with illustrative clinical vignette. \\ Updating and most relevant points for clinical practice
}

\author{
Costa Barney V, Jaramillo Parra $P,{ }^{2}$ Luna Meza A. ${ }^{3}$
}

Internista, Gastroenteróloga, Unidad de Endoscopia Digestiva,
Gastroadvanced - Clínica Palermo, Bogotá, Colombia. Hospital
Universitario San Ignacio, Pontificia Universidad Javeriana.
Bogotá, Colombia.
${ }^{2}$ Internista, Residente de Endocrinología, Hospital Universitario
San Ignacio, Pontificia Universidad Javeriana. Bogotá, Colombia.
${ }^{3}$ Internista, Pontificia Universidad Javeriana. Bogotá, Colombia.

Autor de correspondencia: Valeria Atenea Costa

Correo electrónico: ateneacosta.82@gmail.com

Fecha de recepción: $12 / 05 / 2020$

Fecha de aceptación: 30/11/2020

\section{Resumen}

La gastroparesia diabética es una entidad conocida, aunque en oportunidades se torna difícil su manejo con tratamiento médico, por lo que se deben contemplar otras opciones terapéuticas avanzadas. Es una enfermedad que amerita el tratamiento en conjunto entre gastroenterólogo, endocrinólogo y nutricionista.

Palabras clave: gastroparesia, gastroparesia diabética, piloromiotomía, gastrectomía, complicaciones de la diabetes.

\section{Abstract}

Diabetic gastroparesis is a known entity, although its management with medical treatment is sometimes difficult, so other advanced therapeutic options should be considered. It is a disease that merits the joint treatment between gastroenterologist, endocrinologist and nutritionist.

Keywords: Gastroparesis; Diabetic Gastroparesis; Pyloromyotomy; Gastrectomy; Diabetes Complications.

\section{Caso clínico}

Hombre de 36 años, natural y procedente de Bogotá, Colombia, con antecedente de diabetes mellitus tipo 1 de 10 años de evolución y con complicación de neuropatía diabética. Venía en tratamiento con 18 unidades de insulina glargina en la noche y 8 unidades de aspart prandial; adicionalmente, tomaba pregabalina para el control sintomático de la neuropatía. Acudió a urgencias por 10 meses de dolor abdominal epigástrico opresivo de intensidad 5/10 no irradiado, asociado con la ingesta de alimentos y con sensación de plenitud posprandial, saciedad precoz y náuseas. Refirió que los síntomas se habían exacerbado en los últimos 10 días, por lo que decidió consultar. El examen físico de ingreso fue normal, excepto por el dolor a la palpación profunda del epigastrio.

Fue valorado por medicina interna en urgencias y solicitaron paraclínicos que incluyeron hemograma, bilirrubinas, transaminasas, fosfatasa alcalina y amilasa, los cuales fueron normales. Adicionalmente, se observaron los electrolitos con hiponatremia leve. Se realizó una ecografía abdominal total que mostraba como único hallazgo anormal una esteatosis hepática. Considerando los síntomas y los antecedentes del paciente, se solicitó una interconsulta al grupo de gastroenterología y una esofagogastroduodenoscopia. El estudio endoscópico como primera medida descartó una obstrucción del tracto de salida del estómago, pero tenía restos de alimentos en la cámara gástrica a pesar del ayuno.

En concordancia a lo anterior, el equipo de gastroenterología decidió iniciar el tratamiento intrahospitalario con metoclopramida, con lo cual el paciente presentó mejoría de los síntomas. Adicionalmente, se solicitó la educación en diabetes por parte del servicio de endocrinología, valoración por nutrición clínica y, al momento del alta, continuar el tratamiento con 
domperidona. Con el fin de confirmar el diagnóstico, se dio orden para la realización de gammagrafía de vaciamiento gástrico para sólidos de 4 horas, la cual tuvo una retención del 30 \%.

\section{Discusión}

La gastroparesia es definida como un síndrome en el cual hay objetivamente un retraso del vaciamiento gástrico en ausencia de una obstrucción mecánica, lo que ocasiona síntomas típicos tales como saciedad temprana, plenitud posprandial, náuseas, vómito, hinchazón y, en algunos casos, dolor abdominal superior (1). Se han descrito múltiples causas y las más frecuentes son la idiopática $(49,4 \%)$, diabetes mellitus (DM; $25,3 \%)$, medicamentos $(22,9 \%)$ y enfermedades autoinmunes $(10,8 \%)$. Un porcentaje alto corresponde a pacientes de sexo femenino y diabéticos tipo 1 (2).

\section{Fisiopatología}

El estómago es un órgano distensible, muscular, altamente vascularizado en forma de bolsa ubicado en el cuadrante abdominal superior izquierdo; es el segmento del tracto gastrointestinal en el que tienen inicio las principales funciones de secreción y digestión del tubo digestivo, aunque su función más importante está dada por la actividad motora gástrica, la cual es controlada por diversos estímulos intrínsecos y extrínsecos, y cuyo principal regulador extrínseco es el nervio vago. Los estímulos intrínsecos provienen del sistema nervioso entérico y son importantes en la coordinación de la función motora gástrica, especialmente en el período interdigestivo. Las neuronas mientéricas también se comunican con las diferentes células del marcapasos digestivo conocidas como células de Cajal.

Existen 3 actividades básicas para que se presente un adecuado vaciamiento gástrico. La primera de ellas es la actividad de reservorio gástrico, la cual tiene como objetivo almacenar el contenido alimenticio para que pueda ser procesado y entregado al duodeno con el fin de no saturar los mecanismos de digestión y absorción por el intestino. La segunda actividad importante es la mezcla y trituración de nutrientes para formar el quimo. En respuesta a la distensión, en la región proximal del estómago se forman ondas de contracción peristálticas con una frecuencia de 3-4 por minuto que alcanzan una intensidad máxima hacia el antro y logran contraer el píloro. Por efecto de esta actividad contráctil, el alimento se desplaza distalmente hasta llegar al píloro, que se encuentra contraído, lo que genera un efecto de retropulsión fundamental para la mezcla, trituración y homogenización del alimento hasta alcanzar la consistencia de quimo. Durante esta contracción, los alimentos que alcanzan la consistencia de quimo alcanzan a pasar por una luz de aproximadamente $3 \mathrm{~mm}$ que deja el píloro, al igual que los líquidos que abandonan el estómago en el período posprandial temprano. El tiempo máximo de actividad motora posprandial es de aproximadamente 120 minutos. Finalmente, la tercera actividad fundamental es el vaciamiento interprandial, el cual se realiza bajo el control del complejo motor migratorio (CMM). La actividad motora del CMM se presenta en forma de ciclos que tienen una duración de aproximadamente 100 minutos y consta de 3 fases: la fase I constituye el $50 \%-60 \%$ del ciclo y se caracteriza por una casi inactividad, solo con algunas ondas contráctiles ocasionales que no generan movimientos propulsivos. En la fase II, que constituye un $20 \%$-30 \% del ciclo, aumenta la frecuencia de las contracciones, pero siguen siendo irregulares y no generan fenómenos propulsivos. Durante la fase III del CMM, que tiene una duración aproximada de 10 minutos, se generan ondas contráctiles propulsivas regulares con una frecuencia de 3 ciclos por minuto; durante esta fase, el píloro permanece totalmente relajado, lo que permite el vaciamiento del contenido gástrico que no alcanzó a ser transformado en quimo (3).

Hay múltiples mecanismos por los cuales se puede presentar gastroparesia diabética. Se describen brevemente los más importantes. La glucosa juega un papel muy importante en el vaciamiento gástrico dado que tiene la capacidad de retrasarlo o acelerarlo. La carga hormonal intestinal y pancreática influye sobre la concentración de glucosa a nivel gástrico y duodenal, por lo que impactan en un adecuado vaciamiento gástrico (4). El complejo incretina (péptido similar al glucagón tipo 1 [GLP-1] y polipéptido inhibidor gástrico [GIP]) es secretado desde las células K y L del intestino delgado en respuesta a los nutrientes gástricos, la glucosa hepática y la insulina, lo que disminuye los niveles de glucosa, estimula la secreción de insulina e inhibe la secreción del glucagón y la motilidad gástrica (5); de esta forma se retrasa la salida del quimo hacia la luz duodenal.

Los pacientes con gastroparesia a menudo tienen neuropatía autonómica; los estudios sugieren que tanto el componente simpático como parasimpático se ven afectados por daños a nivel neuronal. Igualmente, se ha descrito que la hiperglucemia tiene un efecto directo sobre la desmielinización del nervio vago (6). De igual forma, se han descrito otros mecanismos intrínsecos que hacen parte de la fisiopatología de esta enfermedad. El estrés oxidativo desencadenado por la hiperglucemia persistente hace que se disminuyan los niveles de hemooxigenasa-1, la cual se ha demostrado que tiene un papel protector contra el desarrollo de esta entidad (7). Así mismo, estudios tanto en humanos como en animales han mostrado que las imágenes de patología con muestras gástricas evidencian una pérdida de la masa de células de Cajal, las cuales ya se habían presentado como el marcapaso gástrico.

\section{Manifestaciones clínicas}

Los pacientes con gastroparesia diabética se pueden presentar con múltiples manifestaciones. Soykan y colaboradores informaron en su estudio de 146 pacientes con diagnóstico confirmado, que las náuseas estaban presentes en $92 \%$, vómito en $84 \%$, distensión abdominal en 75 \% y saciedad temprana en 60 \% (8). Es fundamental llevar a cabo una historia clínica completa que 
incluya el tipo de diabetes, el tiempo de evolución desde el diagnóstico, el tipo de antidiabéticos utilizados, los antecedentes familiares y la presencia o no de signos de alarma que obliguen a descartar otros diagnósticos diferenciales.

Algunos datos interesantes resaltan que hasta en el $50 \%$ de los pacientes los síntomas inician de forma aguda, el $33 \%$ de los casos refieren que presentan exacerbaciones de los síntomas de forma periódica y el $10 \%$ de los pacientes indica que el comportamiento de su enfermedad es cíclico (9). Un metaanálisis que buscaba la relación de los síntomas típicos y la confirmación de retraso en el vaciamiento por gammagrafía señaló que la distensión abdominal, el dolor abdominal y el vómito presentan los Odds ratio (OR) más elevados (3,7; 5,0 y 6,2; respectivamente) para predecir retardo en el vaciamiento (10).

En un paciente diabético con síntomas típicos de gastroparesia, el diagnóstico se realiza descartando obstrucción del tracto de salida gástrico y documentando el retraso en el vaciamiento por cualquiera de los métodos no invasivos. Es necesario evaluar el vaciamiento gástrico ya que los síntomas por sí solos no confirman el diagnóstico. Medicamentos que retrasen el vaciamiento (opioides, analgésicos, anticolinérgicos y GLP-1) o lo aceleren (metoclopramida, domperidona y eritromicina) deben ser suspendidos como mínimo 48-72 horas.

La gammagrafía de vaciamiento es el estándar de oro para el diagnóstico. Típicamente, se evalúa el vaciamiento mediante alimentos marcados con tecnecio 99 y se obtienen imágenes basales, a 1 hora, 2 horas y 4 horas (11). La Sociedad de Medicina Nuclear y la Sociedad Americana de Neurogastroenterología y Motilidad recomiendan una prueba de 4 horas utilizando clara de huevo, tostadas con mermelada y agua; y para esta combinación se deben utilizar puntos de corte de $\geq 60 \%$ o $\geq 10 \%$ de retención a las 2 y 4 horas, respectivamente, para el diagnóstico (12).

La prueba de respiración con carbono utilizado en el vaciamiento gástrico (GEBT) utiliza un sustrato, generalmente Spirulina marcado con 13C. El alimento sale del estómago, entra al intestino, donde se absorbe, y se metaboliza en el hígado, desde donde entra al pool de bicarbonato corporal y se excreta en forma de dióxido de carbono $\left(\mathrm{CO}_{2}\right)$ en la respiración y, posteriormente, es detectado por un espectómetro de masas (13).

La cápsula de transmisión inalámbrica no digerible (Medtronic Inc.) transmite datos de $\mathrm{pH}$, presión y temperatura a intervalos regulares a un receptor portátil que usa el paciente. El paso de la cápsula desde el antro a través del píloro hacia el duodeno es identificable por un cambio brusco de un pH gástrico ácido a un pH duodenal alcalino asociado con una explosión de contracciones. Un estudio pivotal encontró una buena correlación entre este método diagnóstico y la gammagrafía de vaciamiento (14).

Finalmente, teniendo en cuenta que para el diagnóstico es indispensable descartar obstrucción del tracto de salida gástrico, la endoscopia de vías digestivas altas (EVDA) es útil porque detecta algunos hallazgos indirectos de retardo en el vaciamiento; algunos pacientes muestran restos de comida retenida en el estómago después de 6 horas de ayuno (Figura 1).

\section{Tratamiento}

Existen 4 principios que Kumar y colaboradores señalan como las metas terapéuticas en la gastropatía diabética (GD): restaurar el estado nutricional, reestablecer la hidratación, controlar los síntomas y permitir un mejor control glucémico de la DM (15). Todo lo anterior conlleva a mejorar la calidad de vida de los pacientes (Figura 2) (9).

Claramente, al presentarse la gastropatía diabética se produce un estado de riesgo nutricional, pobre aporte calórico y deficiencia de vitaminas y minerales, motivo por el cual, según la gravedad, las opciones terapéuticas serán diferentes (1). Aunque la evidencia científica no es abundante, el abordaje empírico y fisiopatológico hace que las recomendaciones partan del hecho de necesitar comidas con bajo contenido graso y fibras, ya que estas suelen retrasar el vaciamiento gástrico. Así mismo, la necesidad de consumir alimentos en porciones pequeñas y fraccionadas hacen que la recomendación en sí sea consumir 4-5 comidas al día bajas en grasas y fibra (1, $16,17)$. Esto hace que alimentos como sopas, batidos, pasta y carnes blancas, entre otros, requieran menor trabajo para el vaciamiento gástrico y sean opciones para el paciente con gastroparesia diabética (18). Lo anterior también recalca que, dentro del pilar del aporte nutricional, el uso de líquidos es un recurso importante por la posibilidad de rehidratar, aportar sales y minerales, y que el vaciamiento gástrico de líquidos no suele estar afectado, aunque es de mal pronóstico nutricional la alteración del vaciamiento de líquidos en la gastroparesia diabética $(1,18)$. Por el contrario, las bebidas carbonatadas (gaseosas) pueden aumentar la distensión gástrica, por lo que su consumo debe evitarse, al igual que el consumo de alcohol y tabaco, porque modifican el vaciamiento gástrico (1).

Se ha demostrado que los estados de hiperglucemia se asocian con un empeoramiento del vaciamiento gástrico o inhiben la contractilidad antral. Aunque es controvertido que el control glucémico a largo plazo mejora los síntomas de gastroparesia diabética, se ha visto que algunos de los medicamentos para el control glucémico como los GLP-1 retrasan el vaciamiento gástrico, mientras que otros como los inhibidores de la dipeptidil peptidasa 4 (iDPP4) tienen un efecto neutro en el vaciamiento $(1,9)$. Otro motivo de su uso controvertido es que, por el retraso en el vaciamiento gástrico, la farmacocinética y farmacodinamia de los antidiabéticos orales puede verse alterada y, de este modo, evitar que el paciente llegue a las metas. Sin embargo, se considera que un mejor control glucémico se asocia con mejor vaciamiento gástrico (17).

El tratamiento por excelencia por muchos años ha sido el uso de procinéticos como la metoclopramida, eritromicina y domperidona (18). Sin embargo, en los casos en que la com- 
Figura 1. Algoritmo diagnóstico de la gastroparesia diabética.

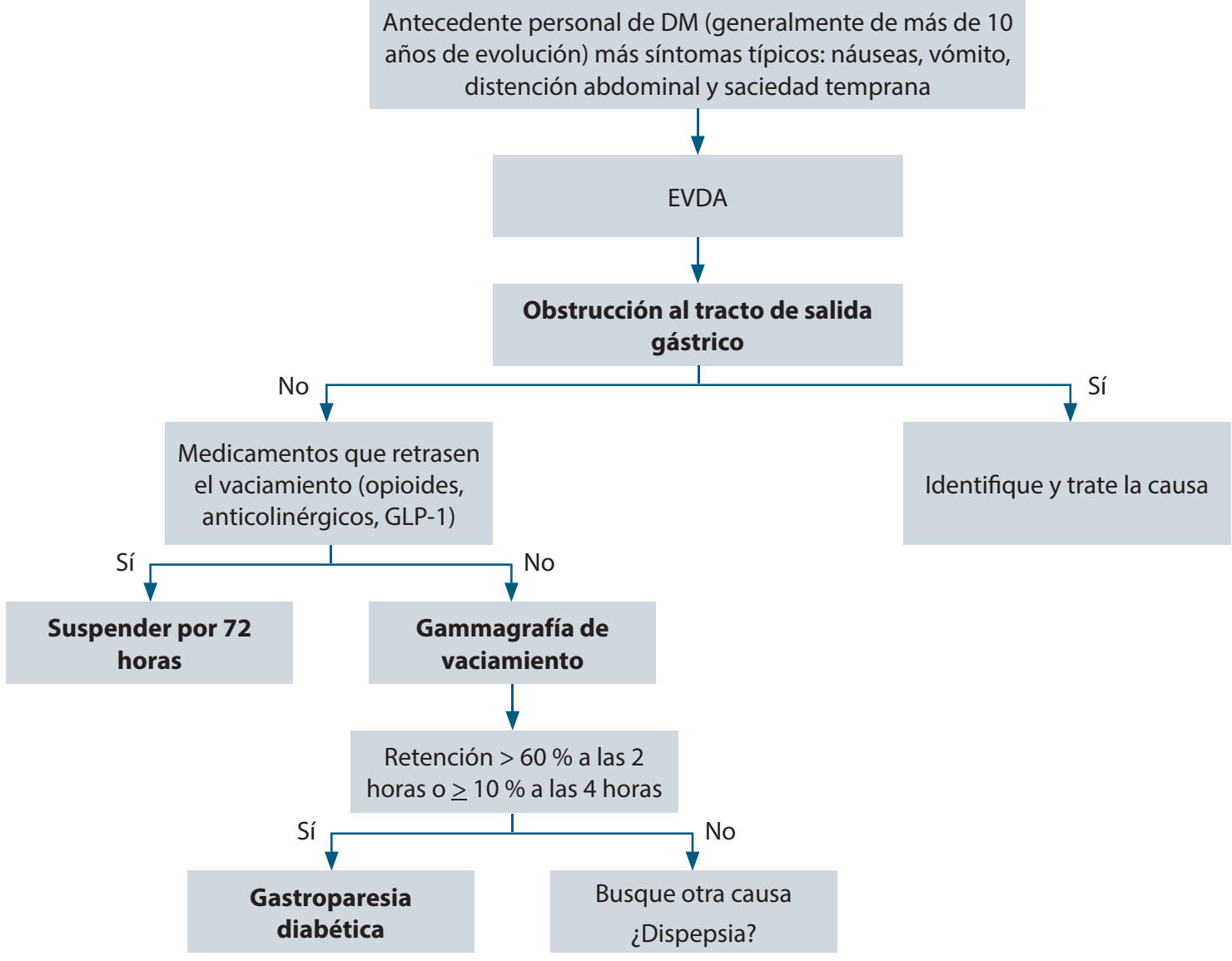

Figura 2. Algoritmo de manejo de la gastroparesia diabética.

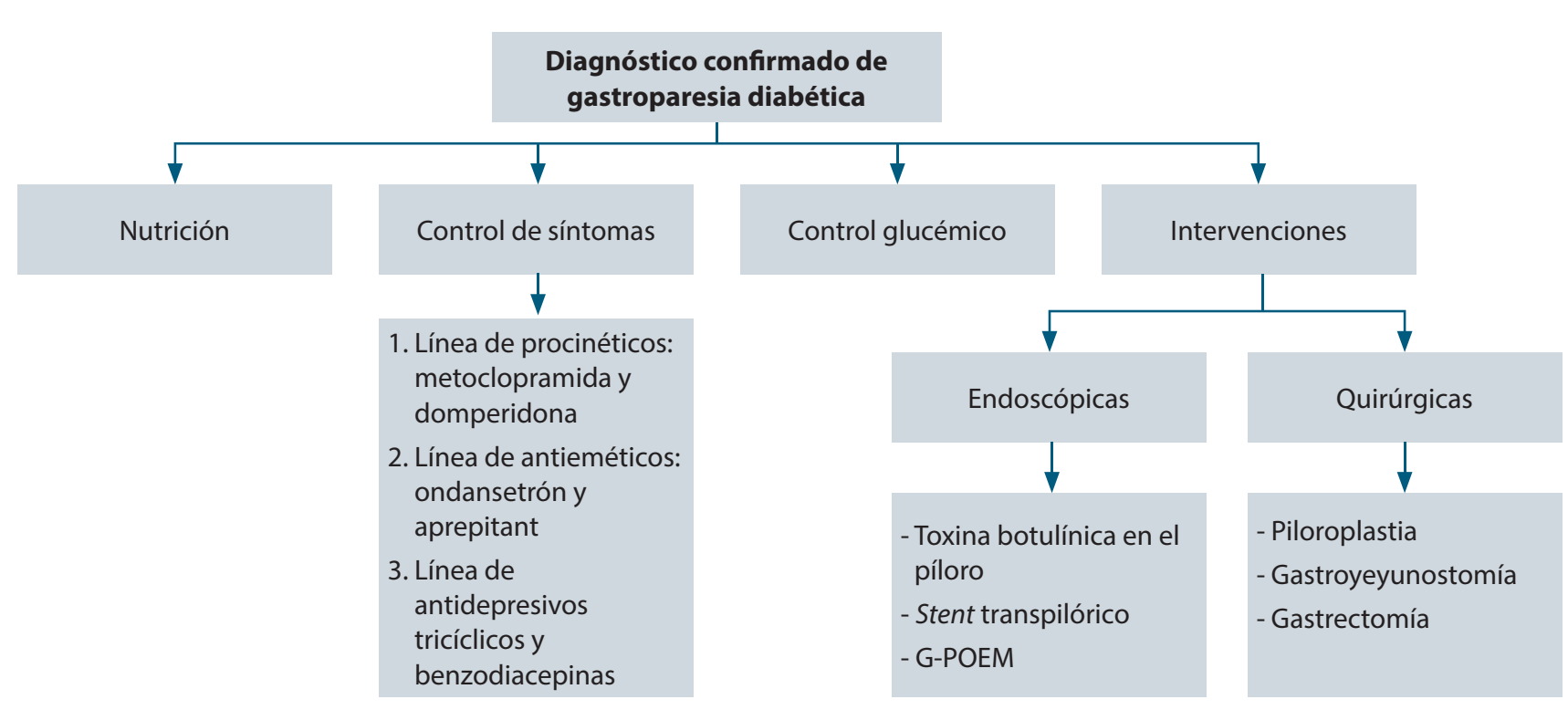

G-POEM: piloromiotomía gástrica endoscópica por vía oral; Qx: quirúrgico. 
binación de estos no es suficiente aparecen como opciones terapéuticas antieméticos como ondansetrón, antidepresivos tricíclicos, benzodiacepinas o las intervenciones endoscópicas o quirúrgicas $(1,15,18)$.

\section{Medicamentos}

\section{Metoclopramida}

$\mathrm{Su}$ principal efecto farmacológico es el antagonismo de los receptores D2 de dopamina. Adicionalmente, aumenta la liberación de acetilcolina en el plexo mientérico, mecanismo a través del cual ejerce su efecto procinético (15). Ricci en 1985 demostró una reducción del 53 \% de los síntomas en los pacientes con gastroparesia diabética al recibir una dosis de $10 \mathrm{mg}$ de metoclopramida preprandial (19). Es necesario tener precaución con los efectos secundarios como discinesia tardía. Este medicamento constituye la primera línea de tratamiento, debe administrarse en la dosis mínima efectiva.

\section{Domperidona}

Es un antagonista de los receptores D2 de dopamina. Ha demostrado disminuir los síntomas de la gastroparesia diabética (20). No atraviesa la barrera hematoencefálica, por lo cual no presenta los efectos adversos generados por la metoclopramida y puede ser utilizado como segunda línea de tratamiento. Es necesario realizar vigilancia electrocardiográfica dado que se han reportado efectos adversos cardiovasculares por la prolongación del intervalo QTc.

\section{Eritromicina}

Este antibiótico del grupo de macrólidos es un agonista de los receptores de motilina y aumenta el peristaltismo gástrico (15). Es utilizado principalmente de forma intrahospitalaria. Sus derivados sintéticos, claritromicina y azitromicina, no han demostrado tener el mismo efecto. Existen otros medicamentos no antibióticos con el mismo mecanismo de acción como mitemcinal, camicinal; sin embargo, hasta el momento la evidencia es controversial sobre el beneficio de estos sobre los síntomas en gastroparesia diabética $(21,22)$.

\section{Aprepitant}

Es un antagonista del receptor de neuroquinina. Su principal uso es el tratamiento de náuseas y vómito por quimioterapia (15). Existen reportes de caso en los cuales los pacientes con gastroparesia diabética que recibieron tratamiento con aprepitant presentaron mejoría de sus síntomas (23). Sin embargo, por el momento no existen estudios específicamente en esta patología.

\section{Agonistas del receptor 5-hidroxitriptamina 4 (5-HT4)}

Aumentan el peristaltismo gastrointestinal a través de la vía colinérgica, liberando acetilcolina en el plexo mientérico (24).
Los medicamentos cisaprida y tegaserod se relacionaron con efectos cardiovasculares adversos y fueron retirados del mercado, aunque un análisis posterior permitió determinar que los eventos cardiovasculares reportados no se relacionaban con el uso de tegaserod (15). La revexeprida tiene un efecto más selectivo. Un ensayo clínico aleatorizado no mostró diferencias en el tiempo de vaciamiento gástrico o los síntomas sugestivos de gastroparesia (25). En esta búsqueda no se encontraron estudios con mosaprida en gastroparesia diabética.

\section{Agonistas del receptor de grelina}

La estimulación del receptor GHS-R1 resulta en un aumento del complejo migratorio y la señalización vagal (15). La relamorelina es un agonista sintético con mayor estabilidad que los péptidos endógenos y ha demostrado mejorar la actividad motora gástrica en pacientes diabéticos tipo 1, así como disminuir los síntomas como náuseas, dolor abdominal, distensión abdominal y plenitud temprana $(26,27)$.

\section{Intervenciones}

La mayoría de las intervenciones se dirigen hacia el píloro, lo cual puede explicar por qué no todos los pacientes se benefician de estos procedimientos. Algunos estudios sugieren que el subgrupo de pacientes con mejor respuesta a los tratamientos intervencionistas comprende a aquellos con evidencia de disfunción pilórica en la electrogastrografía (24).

\section{Toxina botulínica}

Los estudios no han demostrado superioridad en la mejoría de síntomas con respecto a placebo. No se recomienda como tratamiento para la gastroparesia diabética (17).

\section{Stent transpilórico}

Un estudio retrospectivo de 30 pacientes con náuseas y emesis refractaria demostró mejoría significativa de los síntomas en el $75 \%$ de los pacientes. En el $59 \%$ de los pacientes hubo migración del stent a los 146 días, por lo cual se considera una terapia temporal (28).

\section{Estimulación eléctrica gástrica}

Hace referencia a la estimulación del estómago con impulsos eléctricos. Se cree que el mecanismo puede corresponder a acomodación fúndica o estimulación vagal. Ha demostrado disminuir los síntomas asociados a gastroparesia diabética refractaria a 1 año de uso (29).

\section{Cirugía}

\section{Piloroplastia laparoscópica}

El procedimiento más común es la piloroplastia de Heineke-Mikulicz. Los estudios han demostrado una disminución hasta del $75 \%$ de uso de procinéticos, mejoría del vaciamien- 
to gástrico evaluado con gammagrafía y mejoría de los síntomas $(24,29)$. Se considera una opción terapéutica adecuada para la gastroparesia diabética refractaria. Existen diferentes alternativas quirúrgicas para los pacientes con gastroparesia diabética refractaria, entre ellas, la gastroyeyunostomía y la gastrectomía total o subtotal. Estudios observacionales han demostrado que estos procedimientos pueden disminuir los síntomas graves y mejorar la calidad de vida. Sin embargo, debe evaluarse el riesgo de malnutrición y seleccionar a juicio clínico los pacientes candidatos al procedimiento $(1,9)$.

\section{Piloromiotomía gástrica endoscópica por vía oral (G-POEM)}

La pilorotomía endoscópica por vía oral (POEM) se desarrolló inicialmente para el tratamiento de pacientes con estenosis pilórica congénita y la acalasia, y demostró ser una alternativa segura y con tasas de éxito superiores al $90 \%$ a corto plazo (30). Desde 2017 aumentó de forma exponencial el número de casos reportados en quienes se aplicó la G-POEM para el tratamiento de la gastroparesia. Dos series de casos demostraron diferencias en la reducción de síntomas, mientras que otros estudios mostraron resultados contradictorios, especialmente para los pacientes con gastroparesia diabética (9). Los resultados son prometedores. Sin embargo, hacen falta ensayos clínicos aleatorizados. Con base en el principio de las terapias dirigidas a disminuir el piloroespasmo, en 2013 Khashab y colaboradores reportaron el primer caso en el cual se utilizó la G-POEM para el tratamiento de gastroparesia relacionada con diabetes u otras etiologías, con una mejoría de los síntomas diarios que se mantenía 12 semanas después del tratamiento (31).

G-POEM es una técnica que utiliza los principios de endoscopia submucosa en la cual se realiza un túnel submucoso en dirección al píloro, a través del que se realiza una miotomía al anillo pilórico (32). A partir de este momento se han realizado múltiples publicaciones en las cuales esta técnica ha mostrado resultados prometedores con la ventaja de ser mínimamente invasiva. En 2018 Xu y colaboradores presentaron una serie de pacientes asiáticos con gastroparesia refractaria al tratamiento conservador, con una tasa de éxito de 81,25 \%, definido como disminución mayor de 50 \% en el índice del síntoma cardinal de gastroparesia (GCSI), con un seguimiento promedio de 14,5 meses; sin embargo, únicamente el 18,8 \% de los pacientes tenía diagnóstico de diabetes (33). En 2019, Jacques y colaboradores demostraron que la G-POEM mejoraba significativamente los síntomas diarios de pacientes con gastroparesia refractaria (GCSI: 1,3 frente a 3,5; $p<0,001$ ). En este estudio, el $50 \%$ de los pacientes tenía gastroparesia diabética y la incidencia de eventos adversos serios reportados fue inferior al $5 \%$ (34).

Los efectos adversos reportados se encuentran clasificados en tempranos y tardíos. Los primeros corresponden a lesión mucosa, sangrado mayor, enfisema subcutáneo y neumoperitoneo; y los efectos adversos serios se reportaron en menos del $5 \%$ de los procedimientos (34). Los efectos adversos tardíos corresponden principalmente a enfermedad por reflujo gastroesofágico (35). Las contraindicaciones para la realización del procedimiento son: antecedente de radioterapia en la región del esófago y estómago, antecedente de resección extensa de mucosa esofágica o ablación en la zona de interés, enfermedad pulmonar grave, trombocitopenia grave y cirrosis con documentación de hipertensión portal con o sin várices esofágicas (35).

Aún no existe claridad sobre los factores predictores de éxito de esta intervención, aunque el sexo femenino y la presencia de diabetes como etiología de la gastroparesia se han relacionado con el fracaso del procedimiento (36).

\section{Conclusiones}

La gastroparesia diabética continúa siendo un diagnóstico poco frecuente, pero que impacta negativamente en la calidad de vida de los pacientes que la padecen. El médico debe pensar en esta patología y sospecharla para poder realizar un abordaje diagnóstico adecuado y un tratamiento que impacte en el control sintomático de los pacientes. Los diferentes grupos farmacológicos cuentan con ensayos clínicos aleatorizados que respaldan su uso; recientemente, se han descrito múltiples técnicas endoscópicas que resurgen como una alternativa para aquellos pacientes que no responden a las primeras líneas de tratamiento.

\section{Referencias}

1. Camilleri M, Parkman HP, Shafi MA, Abell TL, Gerson L. Clinical Guideline: Management of Gastroparesis. Am J Gastroenterol. 2013;108(1):18-37.

2. Jung $\mathrm{H}$. The incidence, prevalence, and outcomes of patients with gastroparesis in Olmsted County, Minnesota, from 1996 to 2006. J Neurogastroenterol Motil. 2010;16(1):99-100.

3. Rodríguez Varón A, Zuleta J. De la fisiología del vaciamiento gástrico al entendimiento de la gastroparesia. Rev Col Gastroenterol. 2010;25(2):219-225.

4. Phillips LK, Deane AM, Jones KL, Rayner CK, Horowitz M. Gastric emptying and glycaemia in health and diabetes mellitus. Nat Rev Endocrinol. 2015;11(2):112-28.
5. Marathe CS, Rayner CK, Jones KL, Horowitz M. Novel insights into the effects of diabetes on gastric motility. Expert Rev Gastroenterol Hepatol. 2016;10(5):581-93.

6. Krishnasamy S, Abell TL. Diabetic Gastroparesis: Principles and Current Trends in Management. Diabetes Ther. 2018;9(Suppl 1):1-42.

7. Choi KM, Gibbons SJ, Nguyen TV, Stoltz GJ, Lurken MS, Ordog T, et al. Heme oxygenase- 1 protects interstitial cells of Cajal from oxidative stress and reverses diabetic gastroparesis. Gastroenterology. 2008;135(6):2055-64, 2064.e1-2 
8. Soykan I, Sivri B, Sarosiek I, Kiernan B, McCallum RW. Demography, clinical characteristics, psychological and abuse profiles, treatment, and long-term follow-up of patients with gastroparesis. Dig Dis Sci. 1998;43(11):2398404.

9. Bharucha AE, Kudva YC, Prichard DO. Diabetic Gastroparesis. Endocr Rev. 2019;40(5):1318-1352

10. Vijayvargiya P, Jameie-Oskooei S, Camilleri M, Chedid V, Erwin PJ, Murad $\mathrm{MH}$. Association between delayed gastric emptying and upper gastrointestinal symptoms: a systematic review and meta-analysis. Gut. 2019;68(5):804-813.

11. Camilleri M, Iturrino J, Bharucha AE, Burton D, Shin A, Jeong ID, et al. Performance characteristics of scintigraphic measurement of gastric emptying of solids in healthy participants. Neurogastroenterol Motil. 2012;24(12):1076-e562.

12. Pasricha PJ, Yates KP, Nguyen L, Clarke I, Abell TL, Farrugia G, et al. Outcomes and Factors Associated With Reduced Symptoms in Patients With Gastroparesis. Gastroenterology. 2015;149(7):1762-1774.e4.

13. Bharucha AE, Camilleri M, Veil E, Burton D, Zinsmeister AR. Comprehensive assessment of gastric emptying with a stable isotope breath test. Neurogastroenterol Motil. 2013;25(1):e60-9.

14. Kuo B, McCallum RW, Koch KL, Sitrin MD, Wo JM, Chey WD, Hasler et al. Comparison of gastric emptying of a nondigestible capsule to a radiolabelled meal in healthy and gastroparetic subjects. Aliment Pharmacol Ther. 2008;27(2):186-96.

15. Kumar M, Chapman A, Javed S, Alam U, Malik RA, Azmi S. The Investigation and Treatment of Diabetic Gastroparesis. Clin Ther. 2018;40(6):850-861.

16. Tack J, Carbone F, Rotondo A. Gastroparesis. Curr Opin Gastroenterol 2015;31(6):499-505.

17. Horváth VJ, Izbéki F, Lengyel C, Kempler P, Várkonyi T. Diabetic gastroparesis: functional/morphologic background, diagnosis, and treatment options. Curr Diab Rep. 2014;14(9):527.

18. Koch KL, Calles-Escandón J. Diabetic gastroparesis. Gastroenterol Clin North Am. 2015;44(1):39-57.

19. Ricci DA, Saltzman MB, Meyer C, Callachan C, McCallum RW. Effect of metoclopramide in diabetic gastroparesis. J Clin Gastroenterol. 1985;7(1):25-32.

20. Heckert J, Parkman HP. Therapeutic response to domperidone in gastroparesis: A prospective study using the GCSI-daily diary. Neurogastroenterol Motil. 2018;30(1).

21. McCallum RW, Cynshi O; Investigative Team. Clinical trial: effect of mitem cinal (a motilin agonist) on gastric emptying in patients with gastroparesis - a randomized, multicentre, placebo-controlled study. Aliment Pharmacol Ther. 2007;26(8):1121-30.

22. Hellström PM, Tack J, Johnson LV, Hacquoil K, Barton ME, Richards DB, et al. The pharmacodynamics, safety and pharmacokinetics of single doses of the motilin agonist, camicinal, in type 1 diabetes mellitus with slow gastric emptying. Br J Pharmacol. 2016;173(11):1768-77.
23. Fountoulakis N, Dunn J, Thomas S, Karalliedde J. Successful management of refractory diabetic gastroparesis with long-term Aprepitant treatment Diabet Med. 2017;34(10):1483-1486.

24. Navas CM, Patel NK, Lacy BE. Gastroparesis: Medical and Therapeutic Advances. Dig Dis Sci. 2017;62(9):2231-2240.

25. Tack J, Rotondo A, Meulemans A, Thielemans L, Cools M. Randomized clinical trial: a controlled pilot trial of the 5-HT4 receptor agonist revexepride in patients with symptoms suggestive of gastroparesis. Neurogastroenterol Motil. 2016;28(4):487-97.

26. Shin A, Camilleri M, Busciglio I, Burton D, Smith SA, Vella A, et al. The ghrein agonist RM-131 accelerates gastric emptying of solids and reduces symptoms in patients with type 1 diabetes mellitus. Clin Gastroentero Hepatol. 2013;11(11):1453-1459.e4.

27. Lembo A, Camilleri M, McCallum R, Sastre R, Breton C, Spence S, et al. Relamorelin Reduces Vomiting Frequency and Severity and Accelerates Gastric Emptying in Adults With Diabetic Gastroparesis. Gastroenterology. 2016;151(1):87-96.e6.

28. Khashab MA, Besharati S, Ngamruengphong S, Kumbhari V, El Zein M, Stein EM, et al. Refractory gastroparesis can be successfully managed with endoscopic transpyloric stent placement and fixation (with video). Gastrointest Endosc. 2015;82(6):1106-9.

29. Hibbard ML, Dunst CM, Swanström LL. Laparoscopic and endoscopic pyloroplasty for gastroparesis results in sustained symptom improvement. J Gastrointest Surg. 2011;15(9):1513-9.

30. Inoue H, Minami H, Kobayashi Y, Sato Y, Kaga M, Suzuki M, et al. Peroral endoscopic myotomy (POEM) for esophageal achalasia. Endoscopy. 2010;42(4):265-71.

31. Khashab MA, Stein E, Clarke JO, Saxena P, Kumbhari V, Chander Roland B, et al. Gastric peroral endoscopic myotomy for refractory gastroparesis: first human endoscopic pyloromyotomy (with video). Gastrointest Endosc. 2013;78(5):764-8.

32. Chung H, Khashab MA. Gastric Peroral Endoscopic Myotomy. Clin Endosc 2018;51(1):28-32.

33. Xu J, Chen T, Elkholy S, Xu M, Zhong Y, Zhang Y, et al. Gastric Peroral Endoscopic Myotomy (G-POEM) as a Treatment for Refractory Gastroparesis: Long-Term Outcomes. Can J Gastroenterol Hepatol. 2018;2018:6409698.

34. Jacques J, Pagnon L, Hure F, Legros R, Crepin S, Fauchais AL, et al. Perora endoscopic pyloromyotomy is efficacious and safe for refractory gastroparesis: prospective trial with assessment of pyloric function. Endoscopy. 2019;51(1):40-49.

35. Cho YK, Kim SH. Current Status of Peroral Endoscopic Myotomy. Clin Endosc. 2018;51(1):13-18.

36. Gonzalez JM, Benezech A, Vitton V, Barthet M. G-POEM with antro-pyloromyotomy for the treatment of refractory gastroparesis: mid-term follow-up and factors predicting outcome. Aliment Pharmacol Ther. 2017;46(3):364-370 
